\title{
The Effect of Physical Activity on Cardiovascular Markers
}

\author{
Mehran Ghahramani (iD) ${ }^{1,}$, Sara Karbalaeifar (iD) ${ }^{2}$ and Abdolhamid Zokaei (iD ${ }^{3}$ \\ ${ }^{1}$ Department of Exercise Physiology, Gilan-E-Gharb Branch, Islamic Azad University, Gilan-E-Gharb, Iran \\ ${ }^{2}$ Department of Physical Education University of Tehran, Kish International Campus, Kish, Iran \\ ${ }^{3}$ Imam Ali Hospital, Kermanshah University of Medical Sciences, Kermanshah, Iran \\ "Corresponding author: Assistant Professor, Exercise Physiology, Department of of Exercise Physiology, Gilan-E-Gharb Branch, Islamic Azad University, Gilan-E-Gharb, Iran. Tel: \\ +98-9188342771, Email: mehran.physiology@gmail.com
}

Received 2019 April 22; Revised 2019 September 14; Accepted 2019 September 15.

\begin{abstract}
Context: The purpose of this study was to investigate the research framework on the effect of physical activity on cardiovascular markers (C-reactive protein (CRP) and Homocysteine (HCY)), and to conduct an optimal compilation to present better information from previous studies.

Evidence Acquisition: In this study, a number of articles were searched in specialized databases and 30 articles were selected based on entry and exit criteria. Then the responses to one bout of aerobic activity and one bout of resistance activity and adaptability with aerobic training and resistance training were studied.

Results: Various studies have shown a reverse and significant relationship between regular physical activity and inflammatory indices, and reported that those who are physically more active and have better physical fitness have lower levels of inflammatory indices.

Conclusions: The present study showed that regular physical activity has a beneficial effect on the prevention and treatment of coronary artery disease. Regarding the anti-inflammatory effects of physical activity, regular exercise and regular physical activity play an important role in reducing inflammatory indices, and aerobic exercises can be a useful and appropriate strategy for coping with inflammatory and cardiovascular risk factors.
\end{abstract}

Keywords: Physical Activity, Cardiovascular Markers, Homocysteine, C-Reactive Protein

\section{Context}

Cardiovascular diseases are one of the leading causes of mortality in today's world. Cardiovascular diseases are responsible for one third of deaths in people over 35 years of age. About $45 \%$ of deaths in the developed societies and $25 \%$ to $45 \%$ of deaths in the developing countries are due to cardiovascular diseases. In Iran, cardiovascular diseases account for $46 \%$ of deaths, which is 369 people per day (1). The most common cause of heart disease is coronary artery bypass grafting. The main cause of coronary artery disease is atherosclerosis or clogging of the arteries by depositing lipid and accumulation of lymphocytes in the area, and blood clots in the damaged endothelium (the inner surface of blood vessels). As a result, this process involves the formation of fibrous-lipid plaques that increase as age increases, causing narrowing of the vein and thickening of the intima layer, and eventually complete vein cramps (1).

One of the manifestations of the phenomenon of atherosclerosis is coronary artery disease. Atherosclerosis is referred to as hardening or stiffening of the arteries. Low density lipoprotein particles penetrate the artery wall.
Oxidized cholesterol initiates active inflammation in the artery wall.

Inflammation is an essential element in the formation of coronary artery disease. The smooth muscle cells of the artery wall are enlarged in response to inflammation and form an inflexible cap on an inflammatory plaque. Plaques narrow the coronary arteries and reduce blood flow. These plaques cause angina (chest pain) which occurs due to the lack of oxygen-rich blood to the heart muscle. The plaques make up the clot (thrombosis) and, as a result, a small blockade turns into a large one. Ultimately, it is the clot that blocks the artery and causes blockade of the artery of the heart muscle cells that require oxygen-rich arterial blood. Increasing homocysteine HCY and CRP and pro-inflammatory cytokines (TNF- $\alpha$-IL6-IL1) beyond optimal and favorable amount are strong predictors of the incidence of atherogenic events associated with cardiovascular risk factors (2).

A group of risk factors for coronary artery disease have been identified. In this paper, the C-reactive protein (CRP) as an inflammatory marker and homocysteine (HCY) as a 
risk factor for heart disease which independently show the multiple cardiac problems of atherosclerosis risk and the effect of physical activity are investigated.

\section{Homocysteine (HCY) as a Cardiac Risk Factor}

One of the most important modifiable risk factors is homocysteine (HCY) (with a molecular weight of 135.2 $\mathrm{DL})$. Homocysteine is a sulfur-containing amino acid that is produced during methionine metabolism and converted to cysteine and methionine via the Beta Synthase/Tetrahydrofolate Reductase/MethyI/Cystathionine enzymes during this metabolism. High levels of homocysteine in the blood can directly damage the lining of coronary arteries and are likely to cause atheroma, and expand formation of blood clots (1). If the production of homocysteine increases or its metabolism is disrupted, its concentration increases in the cell and it enters extracellular fluids and provides the basis for the emergence of coronary artery disease (2). High levels of homocysteine in the blood plasma can cause atherosclerosis in three ways: damage to the interior arterial wall, interference with blood clotting agents, and oxidation of low density lipoproteins (3). Homocysteine along with TNF- $\alpha$ has a cellular damage effect by creating free radicals and as an inducer of apoptosis (scheduled or physiologic cell death) (3). Clinical studies suggest that the increase in homocysteine in the amount of 5 micromoles per liter is similar to an increase in total cholesterol of $20 \mathrm{mg} / \mathrm{dL}$. Many studies have shown that the relationship between total homocysteine levels and atherosclerosis is even stronger than the association between atherosclerosis and cholesterol $(4,5)$. Homocysteine catabolism disorders cause its accumulation in the bloodstream and effectively initiate inflammatory and atherosclerotic processes (6). One of the mechanisms through which homocysteine makes its pathologic effects is the increase in oxidative stress that may be produced for other causes, including physical activity in cells (2). In other words, homocysteine is self-oxidised in plasma, thereby exacerbating oxidative stress by elevating production of reactive oxygen species that causes damage to endothelial cells and LDL oxidation (6). Several factors affect the concentration of serum total tHCY homocysteine, including genetics, smoking, hypertension, creatine levels, total cholesterol and serum protein, and nutritional factors such as vitamin B12, B6, and folate deficiencies (1).

\section{Inflammatory Marker of CRP as a Predictor of Car- diovascular Risk}

One of the main ingredients of the process index is the acute phase response in the blood of the C-reactive pro- tein or CRP, which is part of the plasma that is made in the liver and its production is responsive to inflammation or tissue damage. The blood levels of CRP in healthy people are very low, but it increases in blood with any inflammation in each part of the body. Therefore, CRP represents the body's response to inflammation and is a good marker of the presence of inflammation (7).

The C-reactive protein is a member of the pentraxins family, which consists of five subunits of 23 kilodaltons. It is derived from the liver and endothelium of the coronary arteries and by increasing the emergence of PAI 1, reducing nitric oxide activity, stimulating LDL consumption by macrophages can increase the risk of atherosclerosis (8). Measuring CRP is the best test for arterial inflammation and its measurement can predict heart disease. The blood level of $1 \mathrm{mg}$ per liter of CRP represents a low risk of heart disease. The values between one to three milligrams per liter indicate a moderate risk and values above 3 milligrams per liter indicate a high risk. People with the highest levels of CRP are 7 times more likely to develop cardiovascular disease than those with the lowest CRP levels. Recent studies indicate that CRP is a stronger marker in predicting cardiovascular events than LDL (9).

Epidemiological studies suggest that increased serum homocysteine (HCY) and C-reactive protein are independently associated with the risk of coronary artery disease (7). Plasma levels of CRP are closely correlated with obesity, diabetes and metabolic syndrome (10). Studies have also shown that the inflammatory system is increasing in obese people and is more likely to be affected by cardiovascular disease (7). Obviously, obesity triggers a chronic inflammation and increases the secretion of pro-inflammatory cytokines from adipose tissue during obesity, which includes TNF- $\alpha$ and its soluble receptors (TNFR1 and TNFR2) and IL6. These cytokines cause the liver cells to secrete CRP. Therefore, in such individuals, the level of CRP rises, and weight loss reduces CRP, TNF- $\alpha$, IL8 and increases the insulin sensitivity and adiponectin (11).

\section{The Effects of Physical Activity}

\subsection{Response to a Single Bout of Aerobic Activity}

Three important factors affecting the level of homocysteine changes after a single bout of physical activity are method (type), intensity and duration of exercise (9). Through reactions methionine is converted to homocysteine during its metabolism. The produced homocysteine has three fates: (1) Through reactions involving betaine consumption and the production of dimethyl glycine, it is again converted to methionine. (2) In the pathway of remethylation, in which folic acid and vitamin B12 act as a cofactor, it is converted to methionine. (3) During reactions, the trans-sulfuration pathway turns into cysteine 
amino acids. Since severe exercise is dependent on the phosphogenic system, the production of required creatine in the body results in an increase in homocysteine (9).

Gaume et al. showed that there is an inverse relationship between homocysteine and maximum oxygen consumption $\left(\mathrm{VO}_{2 \max }\right)$ (an important indicator in the estimation of aerobic power and cardiovascular fitness) in women, while there is no inverse relationship in men (12). Gelecek et al. studied the effect of one single bout of aerobic exercise program on the level of homocysteine in 61 healthy and unhealthy active adults aged 19 - 24 years and showed that plasma homocysteine levels increased immediately after one single bout of physical activity (13). Cheng et al. showed that a fixed cycling program with $80 \%$ of $\mathrm{VO}_{2 \max }$ for 30 minutes had no effect on the level of homocysteine in men 18 to 22 years of age (14).

Nikbakht et al. investigated the relationship between physical activity and serum homocysteine concentrations in active and inactive men and men with coronary artery disease and concluded that physical activity reduces the level of homocysteine in these groups, but it was not significant (6). Murtagh et al. selected 50 inactive and overweight men aged 45 to 50 years to determine the effect of a walking session on the levels of CRP and IL6. The subjects performed at 60 to $70 \%$ of maximum heart rate for 45 minutes. The results showed that the level of CRP did not change. In the studies conducted, the intensity of the type of exercise, the duration of exercise, the amount of muscle damage, and the size of muscle involved were effective in CRP response to physical activity. In the exercises with less intensity and duration and hence lower level of involvement of different muscle groups, the CRP response was lower (15).

\subsection{Response to a Single Bout of Resistance Activity}

After resistance training, the level of homocysteine increases due to elevated protein metabolism and the synthesis of creatine for energy production. In the studies that had a single bout of training over 60 minutes and high intensity, homocysteine levels increased. It turns out that high intensity trainings increase the transport of methyl group, which increases homocysteine production. Methionine is initially converted to methionine Sadenosine. When methyl group transmitters increase in any way, including intense exercise, homocysteine production increases. During the exercise, the protein transfer can modify the homocysteine concentration by increasing methionine catabolism. Therefore, protein reduction can increase the amount of homocysteine (7).

The mechanisms related to CRP after one single bout of physical activity are not well defined. Exercise trainings have a dual effect on CRP, which includes the acute effect of one single bout of exercise in increasing CRP for several consecutive days and a chronic interruption in the release of CRP due to the continuation of intense exercise (2). It should be noted that the type of exercise performed has to be noticed. Increased muscle damage may be a reason for increased levels of CRP after exercise. Several studies reported an increase in the amount of CRP after intense anaerobic exercise activity, especially extrinsic exercises (10).

Bizheh et al. investigated the effect of one single bout of circular resistance training on blood homocysteine and C-reactive protein levels. The subjects included 23 healthy and inactive middle-aged men who were randomly assigned to either experimental (12) and control (n $=9$ ). The training program consisted of 10 stations, which was performed at $35 \%$ of a maximum repetition of each subject. Results showed a significant increase in homocysteine and serum hsCRP levels after training in the experimental group $(\mathrm{P}<0.05)$, but these variables did not change significantly in the control group (8).

Van den Burg et al. reported a pathway for increasing serum CRP levels through intense strength training and anaerobic exercise (16). Saghebjoo et al. investigated CRP as a predictor of cardiovascular disease following one single bout maximal activity in the morning and evening. Significant decrease in CRP levels was observed in the post test of the evening exercise compared with the pre-test ( $\mathrm{P}$ $<0.008$ ). However, CRP levels did not significantly change after the morning exercise compared to the pre-test. Highlighting the CRP response, they concluded that it seems the intensive exercise in the afternoon bout compared to the intensive exercise in the morning was less risky and provided more safety (10).

Gaeeni et al. compared the effect of one single bout of acute (short-term) exercise activity on plasma levels of acute phase-reactive protein, alpha-factor and interleukin6 tumor in obese and non-obese boys. The results of the study showed that the CRP values in the obese group were significantly higher in comparison with the normal weight group at all three times (before activity, immediately after activity and one hour after activity). Among the mechanisms of activation of inflammatory pathways through increasing fat mass, are increased stress receptor and the mechanism of oxidative stress in obese people (17).

A cross-sectional study showed that strength training at an intensity of $70 \% 1 \mathrm{RM}$ compared with aerobic exercise at $65 \%\left(\mathrm{VO}_{2 \max }\right)$ along with consumption of vitamin $\mathrm{E}$ significantly increased CRP in the training group (2). 


\section{Adaptation with Exercise}

\subsection{Adaptation with Aerobic Exercises}

Randeva et al. referred to the effect of 6 months of regular aerobic exercise (running on treadmill) in 21 overweight young women on the reduction of total plasma homocysteine (18). Naghi et al. investigated the relationship between regular aerobic physical activity and non-lipid risk factors and the effect of physical activity on the level of homocysteine and C-reactive protein, with high sensitivity to hr CRP, and showed that regular physical activity could significantly reduce blood homocysteine (19).

Taghian et al. studied the effect of 12 weeks of aerobic exercise on aerobic power, body composition, serum levels of homocysteine and CRP in obese women. Results showed that homocysteine levels decreased significantly after 12 weeks of aerobic exercise (7). Bizheh et al. studied the effect of three months of aerobic exercise on hsCRP levels, homocysteine, serum lipids and aerobic power in healthy middle-aged active and inactive men and concluded that after three months of aerobic exercise, aerobic power increased significantly and homocysteine and hsCRP had a significant decrease $(\mathrm{P}<0.5)$. They concluded that significant reduction of homocysteine and hsCRP as two emerging cardiovascular risk factors could be effective in reducing coronary artery disease in healthy middle-aged and inactive men (8).

In Stewart et al. study, the effect of 12 weeks of physical activity in young and old women showed that serum CRP levels decreased in 12 weeks of physical activity (20). Hamedinia et al. examined the effect of 12 weeks of aerobic training on inflammatory factors in obese and lean men. Findings showed a significant reduction in CRP levels only in obese men. The risk of heart attack in people with a CRP of over $2.1 \mathrm{mg} / \mathrm{L}$ is three times more likely than people with a level not higher than $0.55 \mathrm{mg} / \mathrm{L}$. It has also been shown that regular physical activity reduces CRP resting levels (21). Tokmakidis and Volaklis reported the effect of increasing CRP and HCY on the development of coronary artery disease by creating free radicals and triggering planned death (apoptosis) in type 1 diabetes patients. They reported improvement in disease after 8 weeks of moderate aerobic exercise and also reported significant reductions in CRP and HCY (22).

\subsection{Adaptation with Resistance Exercises}

It has been shown that intense and prolonged exercises alter protein metabolism and blood concentrations of certain amino acids and reduce levels of methionine. Reduced availability of methionine increases methionine synthesis. It therefore leads to the accumulation of homocysteine. In this direction, the mechanism of protein transfer increases the concentration of homocysteine during long and intense exercises (7). Zuehlsdorff in a study found that 12 weeks of daily physical trainings (including trainings on muscular strength and endurance and cardiovascular endurance) could significantly reduce the level of homocysteine in the inactive men and women with 32 to 50 years of age (23).

Hubner-Wozniak and Ochocki, in a research conducted on male and female wrestlers, reported that strength and speed trainings in elite wrestlers significantly reduced the levels of CRP and homocysteine HCY (24). Parsian et al. investigated the effect of strength training on serum Creactive protein and plasma fibrinogen levels in untrained young men, and showed that strength training did not significantly reduce serum CRP in the untrained young men (25).

Stanescu et al. reported a decrease in serum CRP levels after 8 and 12 weeks of strength training (26). Naghizadeh et al. investigated the effect of circular resistance training with vitamin $\mathrm{C}$ on variations of homocysteine, $\mathrm{C}$-reactive protein and tumor necrosis factor alpha (TNF- $\alpha$ ) in healthy non-athlete men. The results showed that HCY in the supplementation training group was significantly lower compared to two other groups $(\mathrm{P}=0.04)$, but the level of CRP in the training group was significantly higher than that of the supplementation and control groups. They concluded that circular resistance training with vitamin $\mathrm{C}$ can reduce cardiovascular disease (2).

\section{Discussion}

Rising levels of homocysteine have a major impact on endothelial damage in the vascular cells (coronary arteries). One of the pathophysiological mechanisms of inflammation is the production of cytokines in response to stimuli such as oxidized LDL, macrophages with atherosclerotic plaque and other risk factors such as homocysteine.

According to Mora et al., The effect of exercise on the level of homocysteine can be influenced by the individual's readiness and body response to stress. These findings indicate that type of physical activity and its magnitude have a direct effect on homocysteine changes in blood (27).

Physical activity may help reduce homocysteine by various means, such as improving body composition, increasing the absorption of vitamins in the intestine, increasing the activity of the relevant enzymes, increasing the transport of protein or methyl group, or through other unknown ways. The intensity, type and duration of exercise, age, race, levels of homocysteine and total cholesterol and triglyceride are factors that affect the results. During exercise and aerobic physical activity, due to the increased need for energy and catabolism, amino acids increase, one of which is methionine, resulting in reduced methionine 
concentrations. In addition, regular physical exercises increase the need for metabolic reactions to repair and restore muscle tissue. Because methionine is an amino acid, increasing the need to it for energy and protein synthesis reduces its concentration. Due to the fact that homocysteine is one of the methionine metabolism mediators, reduction of methionine leads to a decrease in homocysteine (21). Aerobic exercises help to reduce the amount of homocysteine and convert homocysteine to methionine and cysteine by increasing the absorption of effective vitamins in the homocysteine cycle, especially the vitamins of group $\mathrm{B}$, and prevent its accumulation in the blood (28). Reduction of oxidative stress caused by regular exercise can also reduce the level of homocysteine (29). Regular sports exercises reduce the oxygen deficiency and reduce the reliance on phosphagen system in the initial minutes of the exercise. Since creatine production in the body occurs during methyl transport reactions in which methionine by converting to homocysteine causes the synthesis of creatine, therefore the synthesis of creatine in the body is associated with an increase in the level of homocysteine. Reduced reliance on phosphagen system during oxygen deficiency can reduce homocysteine (4).

Research has shown that regular physical exercise reduces the oxidized LDL, consequently homocysteine coverts into CRP (7). The C-reactive protein (CRP) is an inflammatory serologic marker that causes a risk of coronary artery disease and is a risk factor that correlates with the severity of CAD; also it is an indicator of inflammation that results in atheroscrotic events such as plaque rupture which is a symptom of the disease (8). The level of CRP is directly related to the risk of insulin resistance, so reduction of CRP increases insulin sensitivity (17). There is a strong reverse relationship between the baseline level of plasma CRP and cardiovascular endurance; in fact, with increased respiratory cardiac endurance, the baseline CRP levels are reduced (9).

The results of the studies showed that inflammatory markers, especially CRP, increased after a session of aerobic exercise as well as intense anaerobic exercise, but this increase was temporary and after an intense exercise session it returns to the baseline state. Acute phase reaction to exercise activity seems to be dependent on the size and type of activity, muscle mass involved and muscle damage (30).

Any factor that reduces inflammatory markers reduces cardiovascular disorders. Findings show that the more aerobic power, the lower the plasma CRP level (31). By increasing the level of maximum oxygen consumption, it can be concluded that aerobic exercises, through the strengthening of the cardiovascular system, reduce the production of pro-inflammatory cytokines from mononuclear cells. It is possible that exercise by reducing the production of cy- tokines from fat tissue and increasing insulin sensitivity reduce inflammation (31). The true mechanism of the effect of regular and controlled strength training on decreasing the amount of CRP is unclear; this is probably a kind of adaptation from regular physical activity that directly and indirectly through controlling the production of glycoprotein CRP in the liver reduces the production of inflammatory cytokines. The increase in CRP can be due to changes in the amount of inflammatory markers in the early acute stages of exercise and the type, severity and duration of the exercise (25). Inflammatory cytokines, including CRP, increase after acute exercise, and some hours and days after exercise return to normal levels (7). In one-session studies, increasing mechanical stress and activating endothelial cells caused by frequent foot-to-ground injuries may be a factor in increasing CRP. Several studies have shown that high levels of CRP are closely related to obesity. It is assumed that the secreted interleukin from fatty acids contributes to the increase in CRP observed in obesity (Interleukin 6 has sometimes increased the CRP synthesis up to 100 times (7). It has also been observed that CRP has a positive and high correlation with fatty tissue measurement indices, such as BMI, waist circumference, and circumference to hip ratio (3). A large correlation between the level of CRP and the early stages of atherosclerosis has been observed in children (7).

\section{Conclusion}

Various studies have shown a reverse and significant relationship between regular physical activity and inflammatory indices, and reported that individuals who are more physically active and have better physical fitness have lower levels of inflammatory indices. Several studies have examined the effect of physical activity on plasma levels of new cardiovascular risk factors and observed that plasma levels of cardiovascular risk factors are affected by intensity, duration and type of exercise protocol (10).

A review of studies shows that at least 8 weeks of regular exercise at various intensities can reduce the $C$ reactive protein (CRP) and reduce atherogenic processes. Physical activity by reducing fat and leptin and increasing adiponectin and insulin sensitivity decreases IL6 and TNF- $\alpha$ and thus reduces CRP. Some studies support the impact of physical activity on cardiovascular risk factors through aerobic activities such as soft and light running, mountain climbing, long-distance walking, swimming, etc. Regular aerobic exercises are effective in improving cardiovascular health and reducing the risk of atherosclerosis by increasing aerobic power, reducing body mass index and reducing cardiovascular risk factors including triglyceride, homocysteine and C-reactive protein. Regular physical activity has a beneficial effect on the preven- 
tion and treatment of coronary artery disease. Physical activity can have a direct positive effect on the cardiovascular system through several mechanisms, such as increased blood volume, decreased viscosity, increased stroke volume, decreased blood pressure, increased antioxidant defenses and altered blood lipids. Also, regarding the antiinflammatory effects of physical activity, regular exercise and regular physical activity play an important role in reducing inflammatory markers, and aerobic exercises can be a useful and appropriate strategy to cope with inflammatory and cardiovascular risk factors.

\section{Acknowledgments}

Thanks and appreciation are offered to all colleagues and researchers who helped us with this article.

\section{Footnotes}

Authors' Contribution: Mehran Ghahramani developed the original idea and the protocol, abstracted and analyzed the data, wrote the manuscript and are its guarantors. All the authors contributed to the development of the protocol, data abstraction and manuscript preparation.

Conflict of Interests: The authors have no conflict of interests to declare.

Funding/Support: This study was not supported by any funding.

\section{References}

1. Sarmar S. [Comparison of homocysteine and folic acid levels and the severity of coronary artery bypass graft in patients with coronary artery disease]. Genet Third Millennium. 2011;9(4). Persian.

2. Naghizadeh H, Akbarzadeh H, Nargooni A. [The effect of circular resistance training with vitamin $\mathrm{C}$ on homocysteine and C-reactive protein and nonsmoking factor of alpha tumor in healthy non-athlete men]. Res Sport Sci. 2010;(26). Persian.

3. Bahram ME, Najjarian M, Sayyah M, Mojtahedi $H$. The effect of an eight-week aerobic exercise program on the homocysteine level and VO2max in young non-athlete men. Feyz. 2013;17(2):149-56.

4. Joubert LM, Manore MM. Exercise, nutrition, and homocysteine. Int J Sport Nutr Exerc Metab. 2006;16(4):341-61. doi: 10.1123/ijsnem.16.4.341. [PubMed: 17136938].

5. Sütken E, Akalin A, Özdemir F, Colak Ö. Lipid profile and levels of homocysteine, leptin, fibrinogen and C-reactive protein in hyperthyroid patients before and after treatment/Hipertiroid hastalarda tedavi öncesi ve sonrasi lipid profili ile homosistein, leptin, fibrinojen ve C-reaktif protein düzeyleri. Dicle Med J. 2010;37(1):1

6. Nikbakht HA, Amirtash AM, Gharouni H, Zafari A. [Relationship between physical activity and fibrinogen concentration and serum homocysteine in active and inactive men with coronary artery disease]. Olympic. 2007;15(2). Persian.

7. Taghian F, Kargarfard M, Kelishadi R. [Effects of 12 weeks aerobic training on body composition, serum homocysteine and CRP levels in obese women]. J Isfahan Med Sch. 2011;29(149):1037-45. Persian.
8. Bizheh N, Ebrahimi Atri A, Jaafari M. [The effect of three months aerobic exercise on levels of hsCRP, homocysteine, serum lipids and aerobic power in healthy and inactive middle aged men]. Daneshvar Med. 2012;19(98):43-50. Persian.

9. Bizheh N, Rashidlamir A, Zabihi AR, Jaafari M. The acute effects of strength training on inflammatory markers predicting atherosclerosis: a study on inactive middle-aged men. Tehran Univ Med J.2011;69(3).

10. Saghebjoo M, Khaliran ZD, Afzalpour ME, Hedayati M, Yaghoubi A Comparison of some prognostic markers of cardiovascular diseases to morning and evening Bruce treadmill test in women.J Birjand Univ Med Sci. 2013;20(3):252-61.

11. Vilarrasa N, Vendrell J, Sanchez-Santos R, Broch M, Megia A, Masdevall $\mathrm{C}$, et al. Effect of weight loss induced by gastric bypass on proinflammatory interleukin-18, soluble tumour necrosis factor-alpha receptors, C-reactive protein and adiponectin in morbidly obese patients. Clin Endocrinol (Oxf). 2007;67(5):679-86. doi: 10.1111/j.13652265.2007.02945.x. [PubMed: 17608757].

12. Gaume V, Mougin F, Figard H, Simon-Rigaud ML, N'Guyen UN, Callier ] et al. Physical training decreases total plasma homocysteine and cysteine in middle-aged subjects. Ann Nutr Metab. 2005;49(2):125-31. doi: 10.1159/000085536. [PubMed: 15860911].

13. Gelecek N, Teoman N, Ozdirenc M, Pinar L, Akan P, Bediz C, et al. Influences of acute and chronic aerobic exercise on the plasma homocysteine level. Ann Nutr Metab. 2007;51(1):53-8. doi:10.1159/000100821. [PubMed: 17356255].

14. Cheng $Z$, Yang $X$, Wang $H$. Hyperhomocysteinemia and endothelial dysfunction. Curr Hypertens Rev. 2009;5(2):158-65. doi: 10.2174/157340209788166940. [PubMed: 20495681]. [PubMed Central: PMC2873778].

15. Murtagh EM, Boreham C, Nevill A, Davison G, Trinick T, Duly E, et al Acute responses of inflammatory markers of cardiovascular disease risk to a single walking session. J Phys Activity Health. 2005;2(3):324-32. doi: 10.1123/jpah.2.3.324.

16. van den Burg PJ, Hospers JE, Mosterd WL, Bouma BN, Huisveld IA. Aging, physical conditioning, and exercise-induced changes in hemostatic factors and reaction products. J Appl Physiol (1985). 2000;88(5):1558-64. doi: 10.1152/jappl.2000.88.5.1558. [PubMed 10797112].

17. Gaeeni AA, Ghasemnian A, Dehkordi KJ, Kazemi AAR, Falahi AA. [Effect of one session of acute (short-term) exercise activity on plasma levels of acute phase-reactive protein, alpha and interleukin- 6 tumor and non-obese subjects]. J Mazandaran Univ Med Sci. 2012;(83):74-8. Persian.

18. Randeva HS, Lewandowski KC, Drzewoski J, Brooke-Wavell K, O'Callaghan C, Czupryniak L, et al. Exercise decreases plasma total homocysteine in overweight young women with polycystic ovary syndrome. J Clin Endocrinol Metab. 2002;87(10):4496-501. doi 10.1210/jc.2001-012056. [PubMed: 12364425].

19. Naghi MR, Alem Aref M, Hedayati M. [The relationship between regular physical activity and non-lipid cardiovascular risk factors and the effect of physical activity on the level of Hcy and highly sensitive Creactive C-protein]. J Islamic Azad Univ. 2012;(22):4. Persian.

20. Stewart LK, Flynn MG, Campbell WW, Craig BA, Robinson JP, Timmerman KL, et al. The influence of exercise training on inflammatory cytokines and C-reactive protein. Med Sci Sports Exerc. 2007;39(10):17149. doi: 10.1249/mss.0b013e31811ece1c. [PubMed: 17909397].

21. Hamedinia MR, Haghighi AH, Ravasi AA. The effect of aerobic training on inflammatory markers of cardiovascular disease risk in obese men. World J Sport Sci. 2009;2(1):7-12.

22. Tokmakidis SP, Volaklis KA. Training and detraining effects of a combined-strength and aerobic exercise program on blood lipids in patients with coronary artery disease. I Cardiopulm Rehabil. 2003;23(3):193-200. doi: 10.1097/00008483-200305000-00006. [PubMed: 12782903].

23. Zuehlsdorff TM. The effects of exercise on homocysteine levels in men and women. University of Nevada. College of Health Sciences; 2003. 
24. Hübner-Woźniak E, Ochocki P. Effects of training on resting plasma levels of homocysteine and C-reactive protein in competitive male and female wrestlers. Biomed Human Kinet. 2009;1:42-6. doi: 10.2478/v10101-009-0011-0.

25. Parsian H, Seyed AS, Ghazalian F, Soheyli S, Khanali F, Shirvani H. [Effects of strength training on C-reactive protein and plasma fibrinogen in unexercised young men]. Ilam Univ Med Sci. 2010;(3). Persian.

26. Stanescu CI, Bartell-Weiss L, Cussler E, Williams DP, Going SB Lohman TG. Effect of resistance training on C-reactive protein in postmenopausal women. Med Sci Sport Exerc. 2004;36(5). S189. doi: 10.1097/00005768-200405001-00904.

27. Mora S, Lee IM, Buring JE, Ridker PM. Association of physical activity and body mass index with novel and traditional cardiovascular biomarkers in women. JAMA. 2006;295(12):1412-9. doi: 10.1001/jama.295.12.1412. [PubMed: 16551713].
28. Dehghan SH, Sharifi GH, Faramarzi M. The effect of eight week low impact rhythmic aerobic training on total plasma homocysteine concentration in older non-athlete women. J Mazandaran Univ Med Sci. 2009;19(72):53-9.

29. Choubineh S, Dabidi Roshan VA, Gaeeni AA. [The effect of two method of continuous and interval aerobic training on hs-CRP levels in wistar rat]. J Movement Sci Sport. 2007;5(9):1-13. Persian.

30. Soheili S, Gaeeni AA, Souri R. [The effect of resistance training on systemic inflammatory indicators in older men]. Olympic. 2009;17(48). Persian.

31. Nouri R, Sheykh Saraf B, Fathollahi Shoorabeh FA, Jalali Dehkordi K, Faramarzi M. [The effect of eight weeks of exercise on fibrinogen changes, C-reactive protein, leukocytes, and cardio respiratory fitness in inactive men]. Olympic. 2013;21(1):7-20. Persian. 